\title{
A MODEL FOR THE TIME DEPENDENT VEHICLE ROUTING PROBLEM WITH TIME WINDOWS UNDER TRAFFIC CONDITIONS WITH INTELLIGENT TRAVEL TIMES
}

\author{
Saeed Khanchehzarrin ${ }^{1, *}$, Maral Shahmizad $^{1}$, Iraj Mahdavi ${ }^{1}$, \\ Nezam Mahdavi-Amiri ${ }^{2}$ And Peiman Ghasemi ${ }^{3}$
}

\begin{abstract}
A new mixed-integer nonlinear programming model is presented for the time-dependent vehicle routing problem with time windows and intelligent travel times. The aim is to minimize fixed and variable costs, with the assumption that the travel time between any two nodes depends on traffic conditions and is considered to be a function of vehicle departure time. Depending on working hours, the route between any two nodes has a unique traffic parameter. We consider each working day to be divided into several equal and large intervals, termed as a time interval of traffic. Here, allowing for long distances between some of the nodes, travel time may take more than one time interval of traffic, resulting in resetting the time interval of traffic at the start of each large interval. This repetition of time interval of traffics has been used in modeling and calculating travel time. A tabu search optimization algorithm is devised for solving large problems. Also, after linearization, a number of random instances are generated and solved by the CPLEX solver of GAMS to assess the effectiveness of our proposed algorithm. Results indicate that the initial travel time is estimated appropriately and updated properly in accordance with to the repeating traffic conditions.
\end{abstract}

Mathematics Subject Classification. 90Cxx.

Received october 7, 2019. Accepted June 27, 2021.

\section{INTRODUCTION}

Vehicle routing problem (VRP) is a well-recognized optimization problem, which refers to a set of problems where a fleet of vehicles concentrated in one or more depots must serve a set of customers each having a certain demand [20]. The aim is to determine a set of routes for capacitated vehicles so that the total cost is minimized and customer demands are satisfied [3]. There are various forms of vehicle routing problems. In the past decade, most researches were concerned with static VRPs [17]. In these problems, parameters including travel time were assumed to be fixed during the time periods. Such problems not being practical, so dynamic problems were introduced for real cases. Most scientific papers studying dynamical problems consider parameters such

Keywords. Vehicle routing problem, time windows, traffic condition, time-dependent.

1 Department of Industrial Engineering, Mazandaran University of Science and Technology, Babol, Iran.

2 Faculty of Mathematical Sciences, Sharif University of Technology, Tehran, Iran.

3 Department of Industrial Engineering, South-Tehran Branch, Islamic Azad University, Tehran, Iran.

*Corresponding author: khanchehzarrin@yahoo.com

(c) The authors. Published by EDP Sciences, ROADEF, SMAI 2021 
as customer requirements and/or the presence of customer to be dynamic [10]. Some studies investigated the routing problem with dynamic travel time with increasing interest in recent years [30]. Indeed, in practical situations, the travel time between two customers is dynamic [15]. For example, due to traffic conditions, VRP with dynamic travel times is most common. Time-dependent vehicle routing problem (TDVRP) in traffic conditions is a dynamic vehicle routing problem. The main purpose in this problem is to minimize the total travel time and the arising costs, assuming that the travel time between any two nodes depends on traffic conditions and is given as a function of the vehicle dispatching time. Various factors can affect travel time and the cost between any two specified nodes. Some of these factors include weather conditions and most importantly traffic conditions. In this case, the most influential factor in choosing the next customer for service is the comparison among the traveling times of routes. In such conditions, the traffic regulations, such as speed limit, are effective in selecting the route for continuing the travel.

A VRP with Time Windows (VRPTW) can be considered as soft time windows (with penalties on earliness and tardiness), or as hard time windows (with earliness and tardiness generally forbidden). Specific problems with hard time windows include security patrol services, bank deliveries, postal deliveries, industrial refuse collections, grocery deliveries, school bus routings, and urban newspaper distributions. Among the soft time windows problems, dial-a-ride problems are important examples. From the practical point of view, for situations in which the vehicles may not have any available place for waiting at customer locations, the soft variant prevails.

Here, we propose a mixed-integer nonlinear programming model for time-dependent vehicle routing problem with time windows (TDVRPTW), with the intention to minimize fixed and variable costs. Hard time window and capacitated vehicles are considered. Available studies on TDVRPTW consider the time interval of traffics being limited to only one interval. Here, we consider the possibility of prolongation of the trip due to long distances between nodes, and allow for the travel time to take more than one interval. It means that our model should allow for the same time interval of traffic conditions to be repeated for many times.

If we know that a customer can receive the products during a certain time window and we also know the traffic conditions, and this time window includes, for example, 2 time intervals of traffic, then depending on the time of other customers, the time to reach this customer as well as other customers can be scheduled with a minimal cost.

Our main contributions are the followings:

- The development of a model for TDVRPTW with intelligent travel times. The term time-dependent is used to express the general state of the problem that considers traffic conditions regarding different times. Since the travel time between the two nodes varies and is determined in accordance with the incurring conditions and the defined time interval of traffic, the word intelligent is used to describe the travel with the emphasis that calculating the time between two nodes is not based on a simple parameter, but it is a little more complicated to be close to reality; and our approach calculates a good estimation for it.

- Solving a challenging variant of the VRP with dynamic times.

- Performing computational experiments and presenting comprehensible analyses of the results.

The remainder of our work is organized as follows. A review of TDVRPTW is given in Section 2. Problem definition and a mathematical model are presented in Section 3. Our solution methodology and its implementation are discussed in Sections 4 and 5, respectively, followed by experimental results. Finally, conclusions and future research directions are provided in Section 7.

\section{Literature REVIEW}

As far as we know, the first reference to a time-dependent travel time model is due to Beasley [4]. The author adapted the savings algorithm in order to take into account two periods of the planning horizon with different values of travel times.

Kok et al. [11] provided a model for TDVRP in which they compared four types of strategies and measured the impact of these factors on traffic congestion according to cost factors such as the number of vehicles and etc. 
They also provided a speed model for each arc which was defined for different periods and showed the hourly traffic peak.

Okude and Taniguchi [19] presented a mathematical model for estimating the time-dependent routing problem solutions, in which the hierarchical traffic network was constructed based on the frequency of road usages.

Sun et al. [26] developed a routing model considering traffic. Two case studies were presented to test the proposed model in Pasadena, USA. Finally, the proposed model was compared with the traditional shortest path algorithm (Dijkstra's algorithm).

Setak et al. [22] presented a time-dependent routing mathematical model that allowed the possibility of existence of one edge in each point. Therefore, the routing problem in this study was presented by considering multi-graph with a FIFO property. Finally, numerical results were obtained using tabu search for 40 samples.

Lai et al. [13] proposed a mixed-integer model for time-dependent vehicle routing problem. They considered multi routes between pairs of nodes with different travel options. They also proposed a tabu search to solve it.

Verbeeck et al. [28] presented a stochastic time-dependent orienteering model for vehicle routing. The uncertainty considered in this study was scenario based. The innovation of this research was to provide a solution method for the rapid and effective solution of the proposed model.

Victoria et al. [29] presented a capacitated vehicle routing model considering time-dependent demand. The proposed mixed integer linear program model was used for humanitarian logistics and the column-generation algorithm was used to solve the model. The model was solved for numerical examples in small and medium scale which indicated the improvement of the solution time significantly.

Soysal and Cimen [24] presented a multi-capacity mathematical model for vehicle routing in the green timedependent mode. In order to solve the problem in large scale, due to the complexity of the proposed model, simulation-based limited dynamic programming (RDP) was used to solve this problem. The numerical example results showed that the proposed heuristic approach has the shorter resolution time than the classical Restricted Dynamic Programming. The proposed model resulted in a $2.3 \%$ reduction in costs and $93.1 \%$ reduction in the resolution time.

Huang et al. [8] investigated operational decision making and time-dependent vehicle routing. The objective function considered in this research was to minimize fuel consumption costs and transportation costs. In this study, two types of time-dependent graphs were considered. The first graph was the customer graph and the second graph was the geographic graph. The customer graphs showed the location of the customers and the geographic graph showed the points of intersection in the transportation network. The problem described was the time-dependent vehicle routing problem with path flexibility, which was modeled under deterministic and stochastic traffic conditions in this study.

Afshar-Nadjafi et al. [1] investigated the multi-depot time-dependent vehicle routing problem. The transportation network was considered as homogeneous and the objective function was intended to reduce the transportation time in this network. Considering the time window for customers and the constraint of the maximum number of vehicles available on each route was of the innovations of this research. The proposed mixed integer programming model was tested for 180 numerical examples and satisfactory results were obtained.

Mancini [16] addressed a model for the single depot TDVRP with a maximum time for routes and service time at customers. The objective was to reduce the total travel time. In this paper, a two phases heuristic model was presented in which in the first phase, considering travel time and congestion level, a solution for the problem was provided using the MRCH method, and it, in the second phase of the heuristic method, was used.

Sabar et al. [21] modeled Dynamic Vehicle Routing Problem (DVRP). Their main objective was to find routes to serve customers at the lowest traveling cost. The time between points was variable due to traffic. Given that these problems are dynamic, the self-adaptive evolutionary heuristic algorithm was used to solve the proposed model. Comparing the results of the proposed algorithm with the genetic algorithm showed a better performance of the proposed algorithm compared to the genetic algorithm.

Avraham et al. [2] presented a mathematical model for the problem time-dependent scenario-based vehicle routing problem. Minimizing route duration along with minimizing tardiness penalties were the main objectives of their model. The initial assumptions of their model were based on the traveling salesperson problem. Their 
proposed model was solved with branch and bound and adaptive large neighborhood search heuristic approaches for 40 customers and 40 scenarios.

Romero et al. [14] developed the time-dependent vehicle routing problem. Their main objective was to minimize the route duration. Considering a piecewise linear function for vehicle movement time was one of their research contributions. Another contribution of their research was consideration of horizon planning being dynamic. Finally, the proposed model was solved for 25, 50 and 100 customers with a column generation approach. The results indicated obtainment of appropriate solutions in reasonable times.

Karoonsoontawong et al. [9] developed a multi-trip time-dependent model for vehicle routing problem. Consideration of soft time windows and overtime constraints were among their contributions. The main objective of their proposed model was to minimize vehicle usage, overtime and early/late soft time window penalty costs. In order to solve the proposed model, the single-trip tour counterpart procedure with post-processing greedy heuristic (ISTTCI-GH) approach was used. The results affirmed an acceptable performance of the proposed approach.

Spliet et al. [25] examined time window assignment vehicle routing problem (TWAVRP) with time-dependent travels. The main contribution of this work was the assignment of time window to customers before determining the amounts of their demands and adhering to this time window after determining the amounts of the demands. The main objective of this study was to minimize transportation costs. In order to solve the proposed model, an exact labeling algorithm and a tabu search heuristic were used.

Donati et al. [5] proposed an ant colony algorithm to solve the vehicle routing problem. The considered objective functions included minimizing two hierarchical objectives: the number of tours and total travel time. Time-dependent local search procedures were presented to solve the proposed model which indicated satisfactory results.

Kritzinger et al. [12] presented an algorithm for solving time-dependent vehicle routing problems with a real example. To solve the proposed model, the Dijkstra algorithm was used. The case study considered was the network of Vienna and the results indicated the validity of the model.

Figliozzi et al. [6] proposed a heuristic algorithm for solving time-dependent VRP considering the time window. The computational results indicated that the average computational time increased proportionally to the square of number of customers.

Seyedhasani et al. [23] presented a mathematical model for vehicle routing with reduced completion times. The innovations considered in this research were as follows: (1) In the optimization, the completion times are considered rather than machine time. (2) The use of the tabu search meta-heuristic algorithm for solving the proposed model. The results of the research indicated $32 \%$ reduction in costs after the implementation of the model.

Gmira et al. [7] presented a mathematical model for minimizing costs in a vehicle routing problem with timedependent travel times. The time dependence for each road network was considered separately. One contribution of the work was considering different shortest paths between any two customers at different times of the day. A tabu search-based heuristic algorithm was used to solve the proposed model. The results of solving the model for 200 nodes and 580 arcs point to a proper performance of the proposed algorithm.

Sun et al. [27] examined time-dependent multi-trip vehicle routing problem. Minimizing the use of vehicles was the most important objective of the proposed model. Also, capacity constraints, maximal working duration constraints, service constraints and time window constraints were some of the issues considered in the model. To solve the proposed model, a Tabu search heuristic approach was employed, using nearest-neighbor heuristic to find an initial solution. The results showed a proper performance of the proposed algorithm.

Zhang et al. [31] examined a Time-Dependent Electric Vehicle Routing Problem. Their most important contribution was considering different speeds in different periods in presence of traffic congestion. Therefore, in order for a vehicle to enter the traffic peak, it needs to pay tolls. An adaptive large neighborhood search (ALNS) heuristic was used to solve the proposed mixed integer model.

Zheng [32] examined a vehicle routing problem model with multiple fuzzy time Windows. Their objectives were to minimize the cost of distributing products and to minimize the mean consumer dissatisfaction. The 
most important contribution of this study was considering traffic flow variation with time. The proposed model was solved with an ant colony optimization (ACO) approach. The results showed an appropriate performance of the proposed algorithm on solving numerical examples.

Considering the available work in the literature, the following research gaps are identified:

(1) Modeling the TDVRPTW problem by considering intelligent travel times.

(2) An effective heuristic method for solving challenging variants of the VRP with dynamic times.

(3) Consideration of urban traffic at different times and time window of customers simultaneously, bringing the problem closer to the real world condition.

Regarding the mentioned literature, there is no TDVRPTW in which traffic conditions and time windows have been considered. Here, we intend to address such a problem and develop an algorithm for its solution and modeling.

\section{Problem definition}

We first present a mixed-integer nonlinear programming (MINLP) model for TDVRPTW. The problem is defined on a complete undirected graph $G=(A, E)$, with $A=\{1,2, \ldots, m$ and $E=\{(i, j) \mid i, j \in A, i \neq j$, with $\{2, \ldots, m$ being the set of customers and the node 1 being the depot. The homogeneous vehicles with the same capacity $Q$ are located at the depot. Each customer $i$ has

(1) A nonnegative demand $\left(r_{i}\right)$,

(2) Time window $\left[a_{i}, b_{i}\right]$, and

(3) Service time $s_{i}$.

Also included in this model are traffic conditions affecting vehicle routing. Therefore, to apply traffic conditions, the working days are divided into intervals having the same length (e.g., hours, days, weeks, etc.), which are called Time Interval of Traffic. Each of these time intervals has specific traffic conditions that are repeated during each time interval. This repeatability feature is used for modeling. For example, an $8 \mathrm{~h}$ working day can be divided into four $2 \mathrm{~h}$ intervals with different traffic conditions, so that the traffic conditions for the passage of vehicles will be favorable during the interval of 8-10 and unfavorable during the interval of 10-12. For each time interval of traffic, due to traffic congestion, the average speed is determined. The initial time, the time between two nodes, is considered in most routing studies as the time between two nodes and is estimated routinely (e.g., the time between two nodes estimated based on the Google map). Here, the time between two nodes in the model is updated during the process, and new times are calculated based on the existing conditions. Therefore, the initial time between the two routes is considered as "initial time", not to be mistaken with the final time between the two routes being determined in the process. Using the departure time and estimation of the initial time between two nodes, the number of time interval of traffics is determined. Because of differences of speed in various time interval of traffics, the initial estimation of travel times in traffic conditions should be updated. Intervals, velocities and the variability of travel times based on departure times are shown in Figure 1. The horizontal axis represents the time interval and the vertical axis shows the change in velocity and travel time based on departure time. It is clearly shown that travel time ranges between 45 and 47.5 based on the departure spanning time between 0 and 100 for the same route.

The assumptions for the problem are:

(1) A fleet of vehicles is available at depot and vehicles are homogeneous with a specified capacity.

(2) The network is non-directional and complete.

(3) Each vehicle starts its trip from a depot and returns to the same depot.

(4) For each customer, a hard time window is defined.

(5) Customer demands are deterministic.

(6) Each customer is visited by only one vehicle.

(7) Each vehicle is assigned to only one route.

(8) The total demand of a customer is satisfied in one visit. 

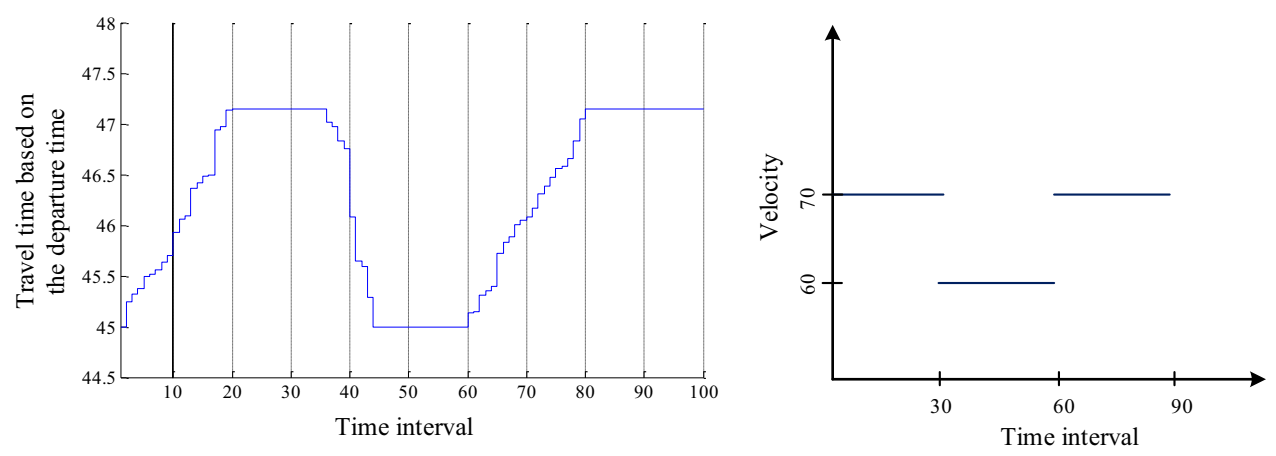

FiguRE 1. Travel time and velocity function.

\subsection{Parameters and indices}

\begin{tabular}{l}
\hline$i, j \in\{1, \ldots, M\}$ \\
$l \in\{1, \ldots, L\}$ \\
$v \in\{1, \ldots, V\}$ \\
$f$ \\
$r_{j}$ \\
$t_{i j l}$ \\
$a_{j}$ \\
$b_{j}$ \\
$g_{l}$ \\
$h_{l}$ \\
$Q$ \\
$s_{j}$ \\
$n \in\{1, \ldots, N\}$ \\
$D_{i j}$ \\
$\mathrm{BM}$ \\
$\varepsilon$ \\
$V e_{i j n}$ \\
$F_{\text {cost }_{v}}$ \\
$V_{\text {cost }_{v}}$
\end{tabular}

Variables:
Set of indices for node (customers and the depot)

Set of indices for the time interval of traffics in a workday

Set of indices for vehicles

Duration of time interval of traffic

Total demand of customer $j$

Estimated time duration between node $i$ and $j$ in time interval of traffic $l$

Earliest time to start the service for customer $j$

Latest time to finish the service for customer $j$

The start time of the $l$ th time interval of traffic

The end time of the $l$ th time interval of traffic

Capacity of a vehicle

Service time for customer $j$

Maximum number of time interval of traffics in all routes

Distance between node $i$ and node $j$

A large number

A small positive number close to zero

Velocity of the vehicle in time interval of traffic $n$ form $i$ to $j$

Fixed cost of vehicle $v$

Variable cost of vehicle $v$

Arrival time to the customer $j$

Departure time from customer $j$

Departure time from depot to customer $j$

Accumulated demand serviced along the route after visiting customer $j$

Auxiliary variable to calculate the updated duration between nodes $i$ and $j$

Updated time duration between nodes $i$ and $j$

Auxiliary variable to calculate the number of time interval of traffics between node $i$ and node $j$ in time interval of traffic $l$ by vehicle $v$

Number of time interval of traffics between nodes $i$ and $j$

1 , if customer $j$ is visited right after customer $i$ in time interval of traffic $l$ by vehicle $v$; 0 , otherwise

Auxiliary variable to calculate the time interval of traffic

Auxiliary variable to calculate the time interval of traffic

1 , if vehicle $v$ is used; 0 , otherwise 


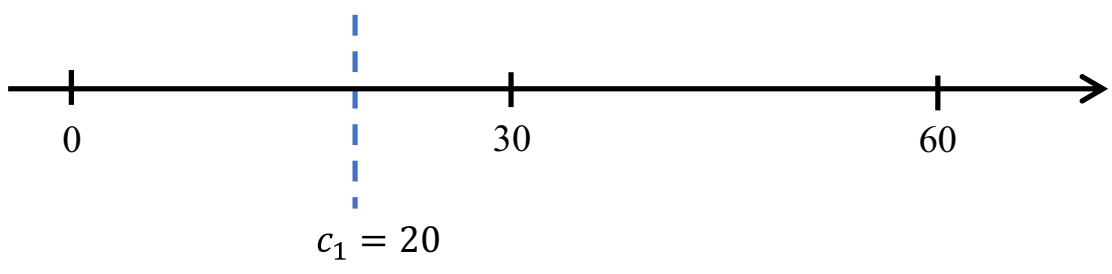

FiguRE 2. Illustrative Example 3.1.

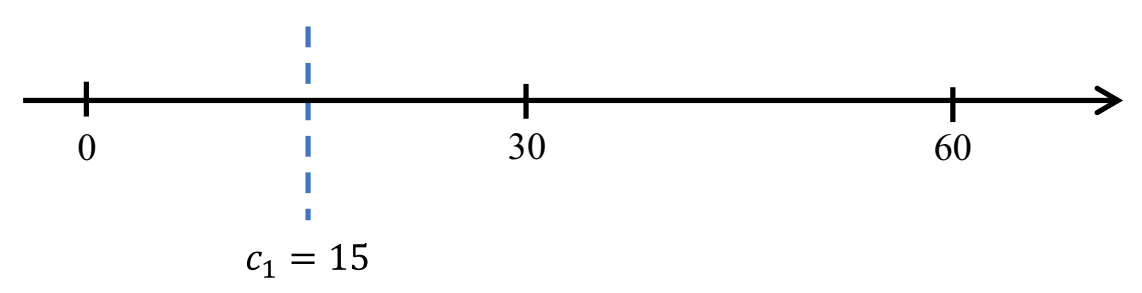

FiguRE 3. Illustrative Example 3.2.

Two auxiliary variables $z_{j l n}$ and $y_{i l n}$ are used to determine the starting time interval of traffic, starting and ending time of the period regarding the move to the next node. In fact, we consider the longest route in terms of time for the whole problem, and determine the maximum number of possible time interval of traffics, $N$. To figure out in which time interval of traffic we started to move, how fast the vehicle is, how much we will remain in the time interval of traffic, etc., we use these two auxiliary variables. For example, suppose $c_{i}=190$. As shown below, $c_{i}$ is between 180 and 210. We use this information to compute the values of $O_{i j l}^{v}, w_{i j}$ and $t_{i j}$. To get this information, we make use of the two variables $z_{j l n}$ and $y_{i l n}$.

For a better understanding of the variables of the functions, the following examples can be helpful. Assume that there are only one vehicle and two customers. The speeds are $70 \mathrm{k} / \mathrm{h}$ and $60 \mathrm{k} / \mathrm{h}$ in the first and second intervals respectively. The distance between the two customers is $17.5 \mathrm{~km}$. The departure time from the first customer is 20 in the first example, and 15 in the second example. The travel time between the two customers at the speed of $70 \mathrm{k} / \mathrm{h}$ is equal to $15 \mathrm{~min}$. The corresponding computations are as follows (Fig. 2).

\section{Example 3.1.}

$$
\begin{aligned}
& t_{121}=15, c_{1}=20, y_{111}=0, x_{121}^{1}=1, o_{121}^{1}=\max \left\{0, \frac{15-(30-20)}{30}\right\}=\frac{5}{30} \\
& w_{12}=\left[\frac{5}{30}\right]+2=2, t_{12}=t_{121}^{\prime}+t_{122}^{\prime}, t_{121}^{\prime}=\min \{30-20,15\}=10 \\
& t_{122}^{\prime}=\min \left\{30, \frac{17.5-11.6666}{60} \cdot 60\right\}=5.83, t_{12}=10+5.83=15.83 .
\end{aligned}
$$

Here, it can be seen that the updated travel time between two customers has changed from 15 to $15.83 \mathrm{~m}$. Since travelling the distance between two customers includes two time intervals $(w=2)$, the speed in the second interval has decreased (Fig. 3).

\section{Example 3.2.}

$$
t_{121}=15, c_{1}=15, y_{111}=0, x_{121}^{1}=1, o_{121}^{1}=\max \left\{0, \frac{15-(30-15)}{30}\right\}=0
$$




$$
w_{12}=[0]+1=1, t_{12}=t_{121}^{\prime}, t_{121}^{\prime}=\min \{30-15,15\}=15, t_{12}=15
$$

In the second example, because travelling the distance between two customers has one time interval $(w=1)$, we see that the time distance between two customers is fixed at 15 and does not change.

\subsection{Mathematical model}

The mathematical model for the problem is:

$$
\min z=\sum_{i=1}^{M} \sum_{j=1}^{M} \sum_{l=1}^{L} \sum_{v=1}^{V} V_{\text {cost }_{v}} t_{i j} x_{i j l}^{v}+\sum_{v=1}^{V} F_{\text {cost }_{v}} y_{v}
$$

s.t.

$$
\begin{array}{ll}
\sum_{i=1}^{M} \sum_{l=1}^{L} \sum_{v=1}^{V} x_{i j l}^{v}=1, & \forall j=2, \ldots, M, i \neq j \\
\sum_{i=1}^{M} \sum_{l=1}^{L} x_{i j l}^{v}=\sum_{i=1}^{M} \sum_{l=1}^{L} x_{j i l}^{v}, & \forall j, \forall v, i \neq j \\
\sum_{v=1}^{V} \sum_{l=1}^{L} x_{i j l}^{v} \leq 1, & \forall j, \forall i, i \neq j \\
\sum_{j=2}^{M} \sum_{l=1}^{L} x_{1 j l}^{v} \leq 1, & \forall v \\
\sum_{i=1}^{M} \sum_{j=1}^{M} \sum_{l=1}^{L} x_{i j l}^{v} \leq \mathrm{BM} y_{v}, & \forall v \\
u_{i}-u_{j}+Q \sum_{v=1}^{V} \sum_{l=1}^{L} x_{i j l}^{v} \leq Q-r_{j}, & \forall j=2, \ldots, M, \forall i, \quad i \neq j \\
r_{j} \leq u_{j} \leq Q, & \\
a_{j} \leq e_{j} \leq b_{j}-s_{j}, & \forall j=2, \ldots, M, \\
e_{i}-e_{j} \leq \mathrm{BM}\left(1-\sum_{v=1}^{V} \sum_{l=1}^{L} x_{i j l}^{v}\right)-\sum_{v=1}^{V} \sum_{l=1}^{L}\left(t_{i j}+s_{i}\right) x_{i j l}^{v}, & \forall j=2, \ldots, M,
\end{array}
$$




$$
\begin{array}{rlrl}
t_{1 j 1}^{\prime}= & \min \left\{\sum_{l=1}^{L} \sum_{v=1}^{V}\left(\sum_{n=1}^{N}\left[\left((n-1) L f+h_{l}\right)\left(1-z_{j l n}\right)\right]-C_{1 j}^{\prime}\right)\right. \\
& \left.x_{1 j l}^{v}, \sum_{v=1}^{V} \sum_{l=1}^{L} t_{1 j} x_{1 j l}^{v}\right\} & \forall j=2, \ldots, M \\
t_{i j n}^{\prime}=\min \left\{f, D_{i j}-\sum_{p=1}^{n-1} d_{i j p} / V_{i j n}\right\}, & \forall i=2, \ldots, M, \forall j, i \neq j, \forall n
\end{array}
$$$$
t_{i j}=\sum_{n=1}^{w_{i j}} t_{i j n}^{\prime}
$$$$
\forall i, \forall j, \quad i \neq j, \forall n
$$

$C_{j}=e_{j}+s_{j}$,

$\forall j=2, \ldots, M$

$-\mathrm{BM} y_{i l n}+\left((n-1) L f+g_{l}\right) \sum_{v=1}^{V} \sum_{j=1}^{M} x_{i j l}^{v} \leq C_{i} \sum_{v=1}^{V} \sum_{j=1}^{M} x_{i j l}^{v}$,

$\forall i=2, \ldots, M, \forall l, \forall n$

$C_{i} \sum_{v=1}^{V} \sum_{j=1}^{M} x_{i j l}^{v} \leq \mathrm{BM} y_{i l n}$

$+\left((n-1) L f+h_{l}\right) \sum_{v=1}^{V} \sum_{j=1}^{M} x_{i j l}^{v}-\varepsilon$,

$\forall i=2, \ldots, M, \forall l, \forall n$

$\sum_{n=1}^{N} y_{i l n}=N-1$

$\forall i=2, \ldots, M, \forall l$

$O_{i j l}^{v}$

$=\max \left\{0,\left(t_{i j l}-\left(\sum_{n=1}^{N}\left[\left((n-1) L f+h_{l}\right)\left(1-y_{i l n}\right)\right]-C_{i}\right) / f\right) x_{i j l}^{v}\right\}$,

$\forall i=2, \ldots, M$,

$\forall j, i \neq j, \forall v, \forall l$

$O_{1 j l}^{v}$

$=\max \left\{0,\left(t_{1 j l}-\left(\sum_{n=1}^{N}\left[\left((n-1) L f+h_{l}\right)\left(1-z_{j l n}\right)\right]-C_{1 j}^{\prime}\right) / f\right) x_{1 j l}^{v}\right\}, \quad \begin{aligned} & \forall i=2, \ldots, M, \\ & i \neq j, \forall v, \forall l\end{aligned}$

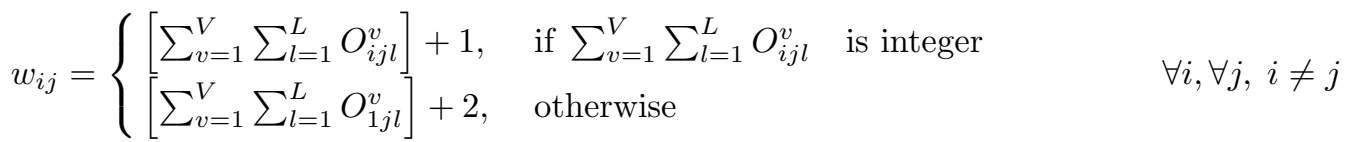

$C_{1 j}^{\prime} \leq e_{j}-\sum_{v=1}^{V} \sum_{l=1}^{L} t_{1 j} x_{1 j l}^{v}+\mathrm{BM}\left(1-\sum_{v=1}^{V} \sum_{l=1}^{L} x_{1 j l}^{v}\right)$

$\forall j=2, \ldots, M$

$C_{1 j}^{\prime} \geq e_{j}-\sum_{v=1}^{V} \sum_{l=1}^{L} t_{1 j} x_{1 j l}^{v}-\mathrm{BM}\left(1-\sum_{v=1}^{V} \sum_{l=1}^{L} x_{1 j l}^{v}\right)$

$\forall j=2, \ldots, M, \forall l, \forall n$

$-\mathrm{BM} z_{j l n}+\left((n-1) L f+g_{l}\right) \sum_{v=1}^{V} x_{1 j l}^{v} \leq C_{1 j}^{\prime} \sum_{v=1}^{V} x_{1 j l}^{v}$,

$\forall j=2, \ldots, M, \forall l, \forall n$

$C_{1 j}^{\prime} \sum_{v=1}^{V} x_{1 j l}^{v} \leq \mathrm{BM} z_{j l n}+\left((n-1) L f+h_{l}\right) \sum_{v=1}^{V} x_{1 j l}^{v}-\varepsilon$,

$\forall j=2, \ldots, M, \forall l, \forall n$ 


$$
\begin{aligned}
& \sum_{n=1}^{N} z_{j l n}=N-1, \\
& x_{i j l}^{v}, y_{v} \in\{0,1\},
\end{aligned}
$$

$$
\begin{aligned}
& \forall j=2, \ldots, M, \forall l, \forall n \\
& \forall i, j, l, \quad v .
\end{aligned}
$$

The objective function (3.6) is to minimize the fixed and variable costs. Constraints (3.7)-(3.10) are common restrictions of the VRP controlling the continuity of the routes. Constraint (3.11) ensures that if the cost of vehicle $v$ is paid off, then it can be used. Constraints (3.12) and (3.13) guarantee that the maximum capacity of each vehicle is not violated. Constraints (3.14) to (3.16) are time window restrictions; the updated time from the model is used in these constraints to control the time windows. In fact, this time is the same time obtained from restrictions (3.17) to (3.20). To consider the service time, a time window constraint is used as constraint (3.14). This ensures that when the service is completed, the time window is not violated. In fact, the arrival and departure times of the customer should fall in the desired time window. Using constraints (3.21)-(3.24) and due to the departure time, traffic conditions for the corresponding time interval of traffic are chosen. Constraints (3.25)-(3.27) determine the number of time interval of traffics between two nodes. Here, using the variables $z_{j l n}$ and $y_{i l n}$, we determine the time interval of traffic in which the moving starts. Then, the starting time $\left(c_{i}\right)$ is subtracted from the upper limit of that time interval of traffic $\left((n-1) L f+h_{l}\right)$, and the variable $O_{i j l}^{v}$ is calculated using the initial time. In fact, since the number of time interval of traffics in a route varies and depends on the starting time of the move, which itself is also a variable, constraints (3.25)-(3.27) show how the number of time interval of traffics is calculated. The variability of the number of time interval of traffics based on the starting time can be explained in a simple example. Assume that in the first time interval of traffic, from time 0 to time 30 , we start at time 10 and the route length is 10 . So, we arrive at the destination at time 20 which corresponds to the time period for this time interval of traffic, and thus we only experience the conditions of one time interval of traffic. But if we move at time 25, we arrive at the destination at time 35 , which corresponds to the second time interval of traffic, falling between 30 and 60, and we experience both the first time interval of traffic and the second time interval of traffic. Constraints (3.28) and (3.29) are used to determine the departure times of vehicles from the depot. When a route is formed from the warehouse to the node $j$, the corresponding relationships are true between the time of leaving the warehouse and the arrival time of the customer based on the time interval between the two nodes. And if the route is not formed, this constraint is relaxed. Constraints (3.30)-(3.32) specify the time interval of traffic in which the movement of each vehicle from the depot is made. In fact, these constraints are to calculate the variable $z_{j l n}$.

Constraint (3.33) gives the binary restrictions.

\subsection{Linearization of the model}

The nonlinearities in (3.6), (3.15), (3.16), (3.28) and (3.29) are linearized as follows. First, we define

$$
\begin{aligned}
& p_{i j l}^{v} \leq \mathrm{BM} x_{i j l}^{v}, \\
& p_{i j l}^{v} \geq t_{i j}-\operatorname{BM}\left(1-x_{i j l}^{v}\right), \\
& p_{i j l}^{v} \leq t_{i j}, \\
& p_{i j l}^{v} \geq 0,
\end{aligned}
$$$$
\begin{array}{llll}
\forall i, & j, & l, & v \\
\forall i, & j, & l, & v \\
\forall i, & j, & l, & v \\
\forall i, & j, & l, & v
\end{array}
$$

Then, the objective function (3.6) and the constraints (3.15), (3.16), (3.28) and (3.29) are written consecutively as follows:

$$
\begin{aligned}
\min z & =\sum_{i=1}^{M} \sum_{j=1}^{M} \sum_{l=1}^{L} \sum_{v=1}^{V} V_{\text {cost }_{v}} p_{i j l}^{v}+\sum_{v=1}^{V} F_{\text {cost }_{v}} y_{v} \\
e_{i}-e_{j} & \leq \operatorname{BM}\left(1-\sum_{v=1}^{V} \sum_{l=1}^{L} x_{i j l}^{v}\right)-\sum_{v=1}^{V} \sum_{l=1}^{L} p_{i j l}^{v}+s_{i} x_{i j l}^{v}, \quad \forall j=2, \ldots, M, \forall i, \quad i \neq j
\end{aligned}
$$




$$
\begin{array}{rlrl}
e_{i}-e_{j} & \geq-\mathrm{BM}\left(1-\sum_{v=1}^{V} \sum_{l=1}^{L} x_{i j l}^{v}\right)-\sum_{v=1}^{V} \sum_{l=1}^{L} p_{i j l}^{v}+s_{i} x_{i j l}^{v}, & & \forall j=2, \ldots, M, \forall i, i \neq j \\
C_{1 j}^{\prime} & \leq e_{j}-\sum_{v=1}^{V} \sum_{l=1}^{L} p_{1 j l}^{v}+\mathrm{BM}\left(1-\sum_{v=1}^{V} \sum_{l=1}^{L} x_{1 j l}^{v}\right) & \forall j=2, \ldots, M \\
C_{1 j}^{\prime} \geq e_{j}-\sum_{v=1}^{V} \sum_{l=1}^{L} p_{1 j l}^{v}-\mathrm{BM}\left(1-\sum_{v=1}^{V} \sum_{l=1}^{L} x_{1 j l}^{v}\right) & \forall j=2, \ldots, M
\end{array}
$$

The nonlinear constraints (3.22), (3.23) and (3.25) are linearized as follows:

$$
\begin{array}{ll}
p 2_{i j l}^{v} \leq \mathrm{BM} x_{i j l}^{v}, & \forall i, j, l, v \\
p 2_{i j l}^{v} \geq C_{i}-\mathrm{BM}\left(1-x_{i j l}^{v}\right), & \forall i, j, l, v \\
p 2_{i j l}^{v} \leq C_{i}, & \forall i, j, l, v \\
p 1_{i j l}^{v} \geq 0, & \forall i, j, l, v \\
p 3_{i j l}^{v}-x_{i j l}^{v}-y_{i l n}+1.5 \geq 0, & \forall i, j, l, n \\
1.5 p 3_{i j l}^{v}-x_{i j l}^{v}-y_{i l n} \leq 0, & \forall i, j, l, n \\
-\mathrm{BM} y_{i l n}+\left((n-1) L f+g_{l}\right) \sum_{v=1}^{V} \sum_{j=1}^{M} x_{i j l}^{v} \leq \sum_{v=1}^{V} \sum_{j=1}^{M} p 2_{i j l}^{v}, & \forall i=2, \ldots, M, \forall l, \forall n \\
\sum_{v=1}^{V} \sum_{j=1}^{M} p 2_{i j l}^{v} \leq \mathrm{BM} y_{i l n}+\left((n-1) L f+h_{l}\right) \sum_{v=1}^{V} \sum_{j=1}^{M} x_{i j l}^{v}-\varepsilon, & \forall i=2, \ldots, M, \forall l, \forall n \\
O_{i j l}^{v}=\max \left\{0,\left(t_{i j l} x_{i j l}^{v}+\sum_{n=1}^{N}\left[\left((n-1) L f+h_{l}\right) p 3_{i j l}^{v}\right]\right.\right. & \\
\left.\left.\quad-\sum_{n=1}^{N}\left[\left((n-1) L f+h_{l}\right) x_{i j l}^{v}\right]+p 2_{i j l}^{v}\right) / f\right\} & \forall i=2, \ldots, M, \forall l, v, j .
\end{array}
$$




\section{Solution methodology: A tabu SeArCh Meta-Heuristic}

Since TDVRPTW was formulated as a mixed-integer linear programming problem (see Sect. 3), commercial optimization software such as GAMS can be used to obtain the optimal solution for small-size instances. In practice, however, the problem is typically quite large and such tools cannot obtain optimal solutions within a reasonable amount of computing time. Thus, here we describe a tabu search meta-heuristic to solve the problem.

Among the meta-heuristics proposed for various vehicle routing problems, tabu search has been shown to be a very effective one [18]. We first give a general structure and then proceed to provide the details of the main components. The search space and initial solution generator are presented in Sections 4.1 and 4.2, respectively. Section 4.3 describes the neighborhood structures. The difference between our presented algorithm and the other algorithms is in the way of satisfying the constraints and satisfying the time window constraint. Based on the time window and vehicle capacity, a feasible solution is to be created, and if an infeasible solution is created, a penalty function is used to enforce feasibility.

\subsection{Search space and handling the constraints}

The search space is thus made up of feasible and infeasible customer assignments. A penalty strategy is considered to handle the constraint. For a given solution $p$, let $c(p)$ denote the total cost of customer assignments, and let $q(p), w(p)$, respectively, denote the total violation of vehicle capacity and customer time windows. The total vehicle capacity violation is computed based on a route considering the value of $Q$, whereas the violation of time windows of customers is equal to $w_{i}=\max \left\{0, e_{i}-\left(b_{i}-s_{i}\right), a_{i}-e_{i}\right\}$, with $e_{i}$ being the arrival time at customer demand $i$. The total violation of time windows is equal to $\sum w_{i}$. Solutions are then evaluated according to the weighted fitness function $f(p)=c(p)+a_{1} q(p)+a_{2} w(p)$, where $a_{1}$ and $a_{2}$ are the penalty parameters.

\subsection{Initial solution}

Here, a constructive heuristic algorithm is used to obtain a satisfactory solution for the described problem. The proposed heuristic consists of three steps: construction of the sequence of customers, SOC, assignment of vehicles, and constructing the routes.

This constructive heuristic procedure is shown as Algorithm 1.

In Section 1-1 of the algorithm, among the customers who have not yet been selected (CL), the one with the smallest $a_{i}$ is selected. Then, customers having overlapping time window with this customer are selected and sorted in increasing order of the time window lengths $\left(b_{i}-a_{i}\right)$ to form RCL in order to determine the customer service priority. When a customer is allocated to a vehicle, and if the vehicle still has capacity, then it is also used for the next customer; this means that priority is given to the vehicle being in use while having remaining capacity. In the provided algorithm, the time window pattern for generating a solution by a heuristic algorithm is used. In this algorithm, the average velocity and time interval of traffic patterns are used to create an initial feasible solution while using the updated time to improve upon the solution.

\subsection{Neighborhood structure}

The solutions in the neighborhood of a given solution are obtained by applying a single-move operation to the current solution. In a single-move operation, we have three operators: swap, reversion, and insertion; all these three operators are used to generate the neighborhood solution. In each iteration, all possible moves of operations for all customers are evaluated and the best one is selected. A tabu move is allowed only when the new solution is feasible and objective function value is better than that of the current best feasible solution. The swap operator for a solution is shown as a neighborhood structure in Figure 4.

There can be two intra-inter route modes for the swap operator. Because the display of the solution is given by a sequence of customers, customer allocation is based on the sequence. So, the first customer in the sequence is allocated to the first vehicle, and based on the created sequence, the other customers are allocated to the first vehicle as long as that vehicle is capable of being allocated. Then, customers in the sequence and not yet allocated to a vehicle are allocated to the second vehicle (considering the capacity constraint). Therefore, the 


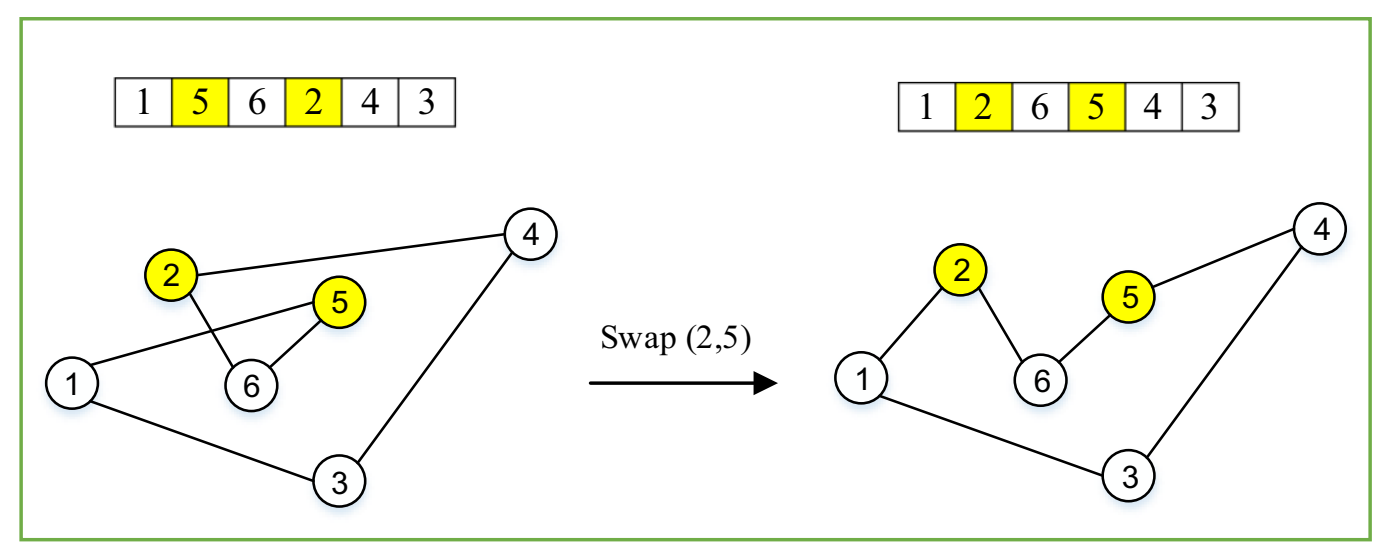

FiguRE 4. Swap operator.

swap operator is applied only to the customer sequence, and the change applied after the sequence may change the sequence of customers in one route or two different routes.

\section{Test PROBlems, PARAMEter tuning, AND implementation}

We first describe the experimental settings in Section 5.1 and then discuss tuning of parameters in Section 5.2, followed by implementation of our algorithm in Section 5.3.

\subsection{Test problems}

Clearly, instance design is important. A number of test problem sets are generated randomly and used here. The dimensions of small and large problem sets are given in Tables 1 and 2, respectively. The model of this study is not a direct development of other studies, and according to our knowledge, this kind of updating the travel time has not been considered in the literature. In fact, random instances have been used.

Due to NP-hardness and complexity of the problem, long running times are needed by exact solution methods. So, we ran the program with a few customers in a small scale to get a solution at a lower cost to validate the problem. We also solved the problem with more customers (up to 9) by GAMS to show that the solution time increases severely, even with a slight increase in the number of customers. Thus, due to the complexity of the problem, we considered a small number of customers. We have solved 10 small scale and 10 large scale problems. The data for large scale examples are presented in the form of random data in a row in Table 2. We used this data to create 10 different problems.

\subsection{Parameter tuning}

Determining the best initial parameter values for an algorithm, called parameter tuning, is crucial for obtaining an effective algorithm performance. Generating a solution of TDVRPTW requires additional knowledge to create good solutions. Therefore, an experimental analysis was conducted to find the most fitting parameters in the algorithm. The parameters and the user defined values are presented in Table 3. In our study, we used the Taguchi method to tune the parameters of the tabu search algorithm.

Here, $n_{\text {Action }}$ is the sum of $n_{\text {swap }}, n_{\text {reversion }}, n_{\text {insertion }}$, which are calculated in accordance with the following relationships. Here, $n$ is the number of problem nodes used to generate a solution (such as the number of genes in a chromosome in the genetic algorithm):

$$
N_{\text {swap }}=n(n-1) / 2
$$


TABLE 1. Initial data for small problems.

\begin{tabular}{|c|c|c|c|c|c|c|}
\hline Sample & $\begin{array}{l}\text { Number of } \\
\text { customers }\end{array}$ & $\begin{array}{l}\text { Number of } \\
\text { vehicles }\end{array}$ & $\begin{array}{l}\text { Vehicle } \\
\text { capacity }\end{array}$ & $\begin{array}{l}\text { Demand } \\
\text { (in order) }\end{array}$ & Time window (in order) & $\begin{array}{l}\text { Service time } \\
\text { (in order) }\end{array}$ \\
\hline \multirow[t]{2}{*}{1} & 4 & 2 & 100 & 20,36 & $(100-170),(170-220)$ & 40,35 \\
\hline & & & & 35,42 & $(135-163),(200-275)$ & 25,40 \\
\hline \multirow[t]{2}{*}{2} & 4 & 2 & 100 & 36,56 & $(100-170),(160-220)$ & 30,25 \\
\hline & & & & 55,42 & $(135-163),(200-275)$ & 25,30 \\
\hline \multirow[t]{3}{*}{3} & 4 & 3 & 100 & 52,56 & $(90-123),(120-150)$ & 30,25 \\
\hline & & & & 55,42 & $(50-100),(200-270)$ & 25,30 \\
\hline & & & & 20,36 & $(100-260),(170-320)$ & 40,35 \\
\hline \multirow[t]{4}{*}{4} & 6 & 2 & 100 & 35,42 & $(135-363),(200-375)$ & 25,40 \\
\hline & & & & 26,29 & $(180-240),(230-320)$ & 15,19 \\
\hline & & & & 20,36 & $(100-260),(170-320)$ & 40,35 \\
\hline & & & & 35,42 & $(135-363),(200-375)$ & 25,40 \\
\hline \multirow[t]{3}{*}{5} & 9 & 4 & 100 & 26,29 & $(180-340),(230-320)$ & 15,19 \\
\hline & & & & 20,26 & $(200-369),(220-300)$ & 22,26 \\
\hline & & & & & $(136-350)$ & \\
\hline 6 & uint $(5,9)$ & uint $(3,5)$ & 100 & uint $(15,20)$ & uint $(100,300)$ & uint $(10,20)$ \\
\hline 7 & uint $(5,9)$ & uint $(3,5)$ & 100 & uint $(20,25)$ & uint $(100,400)$ & uint $(20,25)$ \\
\hline 8 & uint $(5,9)$ & uint $(3,5)$ & 100 & uint $(20,30)$ & uint $(100,280)$ & uint $(20,40)$ \\
\hline 9 & uint $(6,9)$ & uint $(3,5)$ & 100 & uint $(18,25)$ & uint $(100,350)$ & uint $(15,30)$ \\
\hline 10 & uint $(6,9)$ & uint $(3,5)$ & 100 & uint $(18,30)$ & uint $(150,400)$ & uint $(15,40)$ \\
\hline
\end{tabular}

TABLE 2. Initial data for large problems.

\begin{tabular}{|c|c|c|c|c|c|c|}
\hline & $\begin{array}{l}\text { Number of } \\
\text { customers }\end{array}$ & $\begin{array}{l}\text { Number of } \\
\text { vehicles }\end{array}$ & $\begin{array}{l}\text { Vehicle } \\
\text { capacity }\end{array}$ & $\begin{array}{l}\text { Demand } \\
\text { (in order) }\end{array}$ & Time window (in order) & $\begin{array}{l}\text { Service time } \\
\text { (in order) }\end{array}$ \\
\hline 1 & uint $(18,25)$ & uint $(7,10)$ & 100 & uint $(20,30)$ & uint $(100,500)$ & uint $(20,40)$ \\
\hline
\end{tabular}

The range of values set for the parameters of the proposed algorithm, such as the number of iterations and the tabu list coefficients, is determined based on trial and error, and then the parameter tuning is used to select the best values of the parameters in the given intervals. Parameter tuning is not done for the penalty parameter. The amount of this penalty just needs to be large enough, to incur feasibility. Once the amount of penalty is set to be large enough (e.g., it can be a multiple of the value of the objective function, such as OBJ * 1.2), there is no need for further adjustment of the parameter. According to statistical analysis, by using Minitab 17.3.1, the first level of each parameter showed to be the best one (Fig. 5). To tune the parameter with 2 factors and 3 levels, at least 9 tests are needed. Therefore, in order to get the Figure 4, 9 tests are made to set the parameter.

In a Taguchi design, a measure of robustness is used to identify the control factors that reduce variability in a product or process by minimizing the effects of uncontrollable factors (noise factors). Control factors are those design and process parameters that can be controlled. Noise factors cannot be controlled during the production or product use, but can be controlled during experimentation. In a Taguchi designed experiment, noise factors are manipulate to enforce variability and from the obtained results, optimal control factors are set to make the process or product robust, or resistant to variation from the noise factors. Higher values of the signal-to-noise ratio $(\mathrm{S} / \mathrm{N})$ indicate control factor settings that minimize the effects of the noise factors. 
TABle 3. Parameters and user defined values of the algorithms.

\begin{tabular}{lll}
\hline \hline Parameters & Max iteration & Tabu list \\
\hline Levels & 800 & $\operatorname{Round}\left(0.3 * n_{\text {Action }}\right)$ \\
& 1000 & $\operatorname{Round}\left(0.4 * n_{\text {Action }}\right)$ \\
& 1200 & $\operatorname{Round}\left(0.5 * n_{\text {Action }}\right)$ \\
\hline
\end{tabular}
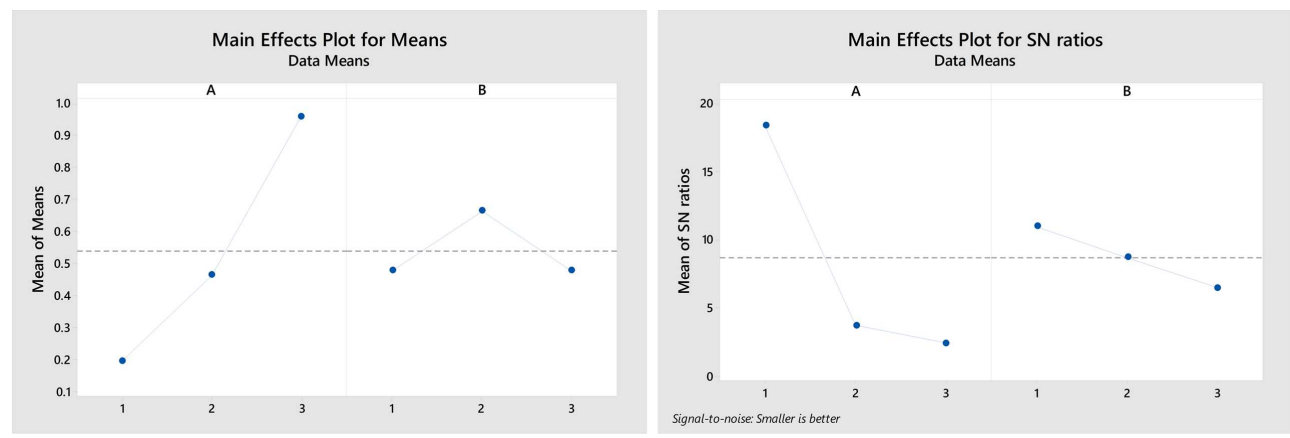

FiguRE 5. Statistical analysis of the Taguchi parameter tuning.

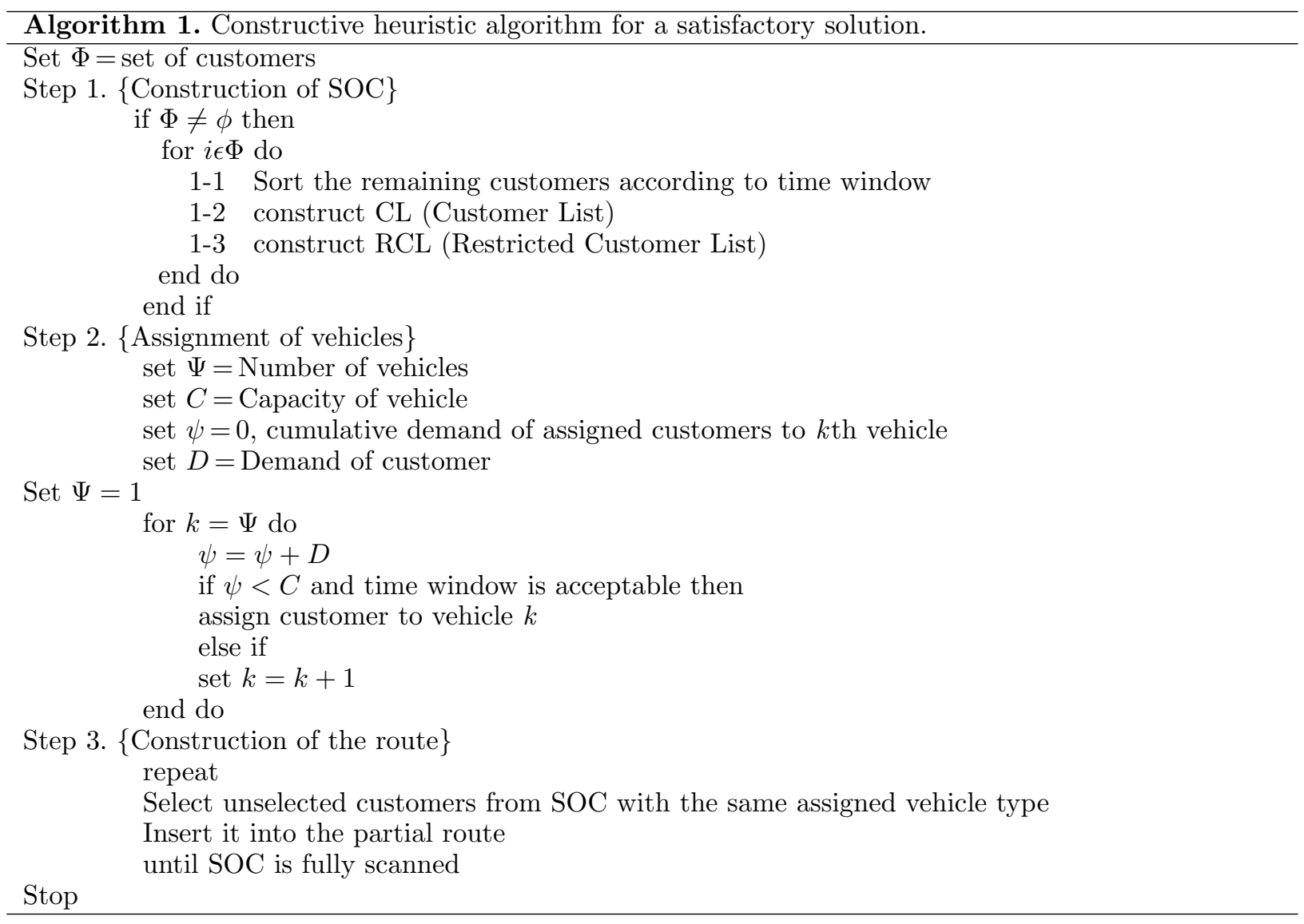


TABLE 4. Computational results for small problems.

\begin{tabular}{lllllllll}
\hline \hline \multirow{2}{*}{ Sample } & \multirow{2}{*}{ Vehicle } & \multirow{2}{*}{ Customers order } & \multicolumn{4}{c}{ GAMS } & \multicolumn{3}{c}{ TS } & \multirow{2}{*}{ Error } \\
\cline { 3 - 7 } & & & Obj & Time (s) & Relative gap & Obj & Time (s) & \\
\hline 1 & 1 & $2-3-5$ & 142.286 & 1 & 0 & 142.29 & 196 & $0.002 \%$ \\
& 2 & 4 & & & & & & \\
2 & - & - & 188.380 & 1 & 0 & 190.1 & 221.3 & $0.91 \%$ \\
3 & - & - & 268.00 & 1 & 0 & 271.29 & 178 & $1.2 \%$ \\
4 & - & - & 190.433 & 361.39 & 0 & 190.89 & 195 & $0.23 \%$ \\
5 & - & - & 384.471 & 380 & 0 & 385 & 197 & $0.13 \%$ \\
6 & - & - & 186.22 & 4503 & 0 & 187.01 & 314 & $0.42 \%$ \\
7 & - & - & 217.84 & 9039 & 0 & 219 & 301 & $0.53 \%$ \\
8 & - & - & 217.87 & 4807.56 & 0 & 221.32 & 235 & $1.58 \%$ \\
9 & - & - & 248 & 14678 & 0.75 & 253.2 & 253 & $2.09 \%$ \\
10 & - & - & 238.54 & 16321 & 0.71 & 240.01 & 266 & $0.61 \%$ \\
\hline
\end{tabular}

TABLE 5. Result for instance 1.

\begin{tabular}{lllllll}
\hline \hline Customer & Demand & Arrival time & Departure time & $\begin{array}{l}\text { Estimated } \\
\text { time duration } \\
\text { to get to the } \\
\text { next node }(t)\end{array}$ & $\begin{array}{l}\text { Updated time } \\
\text { duration to } \\
\text { get } \\
\text { next node }\left(t^{\prime}\right)\end{array}$ & \\
\hline 2 & 20 & 120 & 160 & 10 & 10 & 0 \\
3 & 36 & 170 & 205 & 8.571 & 9.167 & +0.596 \\
4 & 35 & 138 & 163 & 70 & 65 & -5 \\
5 & 42 & 214.167 & 254.167 & 25.714 & 27.361 & +1.647 \\
\hline
\end{tabular}

\subsection{Software-hardware implementation}

The proposed TDVRPTW method was coded using GAMS 24.1.2. The TS method was coded using MATLAB R2014a. All codes were run on a laptop with MS-Windows 8.1 Professional, 1 GB of RAM, and 2.4 GHz Core 2 Due CPU.

\section{Experimental RESUlts}

Here, the obtained results on the test problems are discussed. The results of solving the instances are shown in Table 4. In the last column, the errors of TS in comparison with the results due to GAMS are shown for the objective function. The details of the results of solving the first instance are given in Table 5 and Figure 6 . In the first instance, the value of $f$ is 30 and time interval of traffic and velocities of the vehicles are given in Table 6 . The time interval of traffics defined in Table 6 are considered as hypothetical data for the considered problem. Every problem should have its own data and time interval of traffic. Of these time interval of traffics, traffic conditions and vehicle speed are used in the calculation of time, expressing the concept for intelligent. Here, the penalty amounts are $a_{1}=100$ and $a_{2}=10000$.

According to our obtained results on several instances, it was clear that TS showed a good performance in comparison with GAMS. The maximum of the objective function errors over small size test problems and the mean of errors were less than $2.1 \%$ and near zero respectively, which demonstrated the efficiency and reliability of the proposed algorithm. So, we may trust on the results obtained by the proposed TS on large problem as well. The computing time of the algorithm for this problem is long due to the nature of the problem. This 


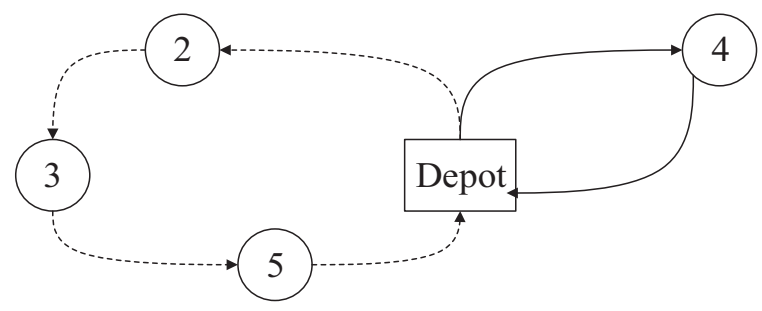

FiguRE 6. Vehicle assignment for instance 1.

TABLE 6. Time interval of traffic intervals and velocities of the vehicles.

\begin{tabular}{lll}
\hline \hline Time interval of traffic & Time & Velocity of vehicle \\
\hline Time interval of traffic 1 & $0-30$ & $70 \mathrm{~km} / \mathrm{h}$ \\
Time interval of traffic 2 & $30-60$ & $60 \mathrm{~km} / \mathrm{h}$ \\
Time interval of traffic 3 & $60-90$ & $70 \mathrm{~km} / \mathrm{h}$ \\
Time interval of traffic 4 & $90-120$ & $60 \mathrm{~km} / \mathrm{h}$ \\
\hline
\end{tabular}

high amount of time may also be due to the MATLAB programming language. Of course, for a professional implementation, better and more efficient programming languages may be used.

In the model, we consider a number of repeating time interval of traffics in different periods. In Table 6 , we defined 4 time interval of traffics having up to 120 time units, for one working day, with the same time interval of traffics repeated at the start of the next day. Because we have two working days, we assumed the beginning of the first day as the starting time or "0", and the end of the second day or "240" as the ending time. To say that the customer wants service on the second long day, we consider the time window of 120 to 240. Determining when to start the move to the customer and which time interval of traffic to incur and, considering the traffic conditions and the starting time interval of traffic, how to update the time relevant to the route length are the concerns of intelligent time. When the relevant time interval of traffic is identified, the speed of that time interval of traffic is used.

The last column in Table $5,\left(t^{\prime}-t\right)$ shows the time changes. It shows whether the real-time is more or less than the estimated time. The difference between the initial time of the two nodes as the problem input and the real time between the two nodes as the output of GAMS are shown. In fact, this shows that whether changing the traffic conditions results in increasing or decreasing the time. Here, the service time for customers $2,3,4$, and 5 is $40,35,25$, and 40, respectively, and based on the arrival time of customers, their departure times are calculated.

Figure 7 displays how to update the time considering two nodes (depot and customer 4) and initial time between the two nodes. The starting time is 73.714 and estimation of travel time is 60 . Two different modes for this route are shown in Figure 7: The first mode is to assume that the traffic conditions are the same along the route, and given that the starting time of the movement is 73.714 and it is located in the third time interval of traffic according to Table 6 , the speed of $70 \mathrm{~km} / \mathrm{h}$ is considered. The time interval between the warehouse and customer 4 is considered to be 60 , regarding the speed of 70 as an input. So, we expect to reach customer 4 at time 133.714. The second mode is that since we know the time to start moving from the warehouse is 73.714 and the time interval of 60 is considered between the two points (warehouse and customer 4), the traffic conditions change after the time 90 and the fourth time interval of traffic occurs when the speed changes to $60 \mathrm{~km} / \mathrm{h}$ from time 90 to 120 and will change again to $70 \mathrm{~km} / \mathrm{h}$ from 120 to 150 (the new course of time interval of traffics starts within 120 to 240 repeating the traffic conditions). This decrease of the speed in the interval of 90 to 120 increases the time for reaching customer 4 ; in other words, the time interval of 60 between the two nodes may 


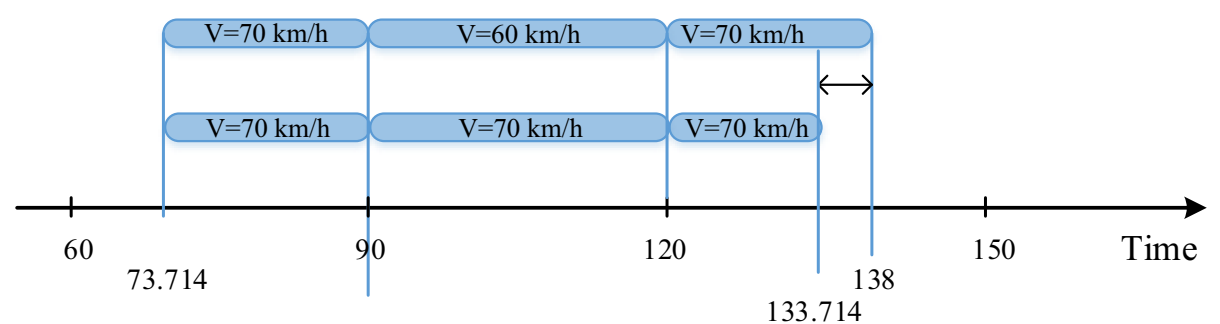

FIgURE 7. Updating the time.

TABLE 7. Computational results for large problems.

\begin{tabular}{lll}
\hline \hline \multirow{2}{*}{ Sample } & \multicolumn{2}{c}{ TS } \\
\cline { 2 - 3 } & Obj & Time (s) \\
\hline 1 & 603.54 & 479 \\
2 & 736.4 & 646 \\
3 & 710.3 & 564 \\
4 & 580.86 & 405 \\
5 & 645.12 & 512 \\
6 & 853.02 & 640.2 \\
7 & 705.6 & 560.98 \\
8 & 597.24 & 484 \\
9 & 633.78 & 523 \\
10 & 699.3 & 516.9 \\
\hline
\end{tabular}

not be considered and a more realistic time should be used in the calculations. A more realistic time is 64.286 , which makes it possible to reach customer 4 at 138, not 133.714. This increase is caused by the reduction of the average velocity of vehicles in time interval of traffic 2 .

Using data in Table 2, ten large test problems are also designed and solved by the TS algorithm. The results are shown in Table 7.

\section{Conclusion}

A mixed-integer nonlinear programming model was presented in the context of time dependent vehicle routing problem with time windows. The aim was to apply the traveling time in the context of more than one time interval of traffic (hours, days, couple of days, weeks, etc.), resulting in resetting the time interval of traffics at the start of each large interval. Having long trips, the model could be applied to companies and transportation services by determining the optimal path and minimizing the total cost. After linearization, the proposed mathematical model was validated using the CPLEX solver of GAMS. We also designed a tabu search algorithm to solve large problems. The model turned to be useful realizing that the travel time changed according to traffic conditions and departure times. After validating the model, the model was solved for 10 examples in small scale. The solution results for the first example were presented in full detail in order to review the time update according to the traffic conditions, and then the manner of updating was shown graphically. The various solved examples indicate that this smart update of the time is done correctly.

\section{Management insights}

The results show that increasing the amount of demand increases the arrival time. For example, increasing the amount of demand from 20 units to 42 units, changes the arrival time from 120 units to 214167 units. 
Therefore, due to demand sensitivity, managers are suggested to have more control over the demand and increase the capacity of vehicles to prevent frequent traffic and environmental pollution. As can be seen, the difference between the initial time and the real time for customer 2 and 3 is 0 and 0.596 units, respectively. Taking a glance at the obtained results in Table 5 and Figure 6, and making a comparison between the estimated time and the updated time, it can be inferred that the proposed model provides more realistic results. Actually, it is worthwhile that we know we are going to reach the destination point at time " $b$ " instead of time " $a$ ". On the other hand, in this model, the time window and traffic conditions are considered simultaneously, an advantage of which being that it turns to make the best decision by the decision maker providing the best schedule and the lowest cost. If managers have complete information for modeling at hand, then optimal decision making may turn to be possible. The results of this study can be used to estimate travel time in distribution of relief goods in disaster. According to Table 4 , the average error of the proposed approach is $0.77 \%$. This indicates the accuracy of the proposed algorithm. Therefore, it can be said that the proposed algorithm can be useful for the development of route scheduling and travel time estimation software.

Acknowledgements. The first three authors thank Mazandaran University of Science and Technology, the fourth author thanks Sharif University of Technology and the fifth author thanks South-Tehran Branch Islamic Azad University for supporting this work.

\section{REFERENCES}

[1] B. Afshar-Nadjafi and A. Afshar-Nadjafi, A constructive heuristic for time-dependent multi-depot vehicle routing problem with time-windows and heterogeneous fleet. J. King Saud Univ.-Eng. Sci. 29 (2017) 29-34.

[2] E. Avraham and T. Raviv, The data-driven time-dependent traveling salesperson problem. Transp. Res. Part B: Methodol. 134 (2020) 25-40.

[3] H.B. Ban, An efficient two-phase metaheuristic algorithm for The Time Dependent Traveling Salesman Problem. RAIRO:OR 53 (2019) 917-935.

[4] J.E. Beasley, Adapting the savings algorithm for varying inter-customer travel times. Omega 9 (1981) 658-659.

[5] A.V. Donati, R. Montemanni, N. Casagrande, A.E. Rizzoli and L.M. Gambardella, Time dependent vehicle routing problem with a multi ant colony system. Eur. J Oper. Res. 185 (2008) 1174-1191.

[6] M.A. Figliozzi, The time dependent vehicle routing problem with time windows: Benchmark problems, an efficient solution algorithm, and solution characteristics. Transp. Res. Part E: Logistics Transp. Rev. 48 (2012) 616-636.

[7] M. Gmira, M. Gendreau, A. Lodi and J.Y. Potvin, Tabu search for the time-dependent vehicle routing problem with time windows on a road network. Eur. J. Oper. Res. 288 (2021) 129-140.

[8] Y. Huang, L. Zhao, T. Van Woensel and J.P. Gross, Time-dependent vehicle routing problem with path flexibility. Transp. Res. Part B: Methodol. 95 (2017) 169-195.

[9] A. Karoonsoontawong, P. Punyim, W. Nueangnitnaraporn and V. Ratanavaraha, Multi-trip time-dependent vehicle routing problem with soft time windows and overtime constraints. Networks Spatial Econ. (2020) 1-50.

[10] M. Keskin, G. Laporte and B. Catay, Electric vehicle routing problem with time-dependent waiting times at recharging stations. Comput. Oper. Res. 107 (2019) 77-94.

[11] A.L. Kok, E.W. Hans and J.M. Schutten, Vehicle routing under time-dependent travel times: the impact of congestion avoidance. Comput. Oper. Res. 39 (2012) 910-918.

[12] S. Kritzinger, K.F. Doerner, R.F. Hartl, G. Y. Kiechle, H. Stadler and S.S. Manohar, Using traffic information for timedependent vehicle routing. Proc.-Soc. Behav. Sci. 39 (2012) 217-229.

[13] D.S. Lai, O.C. Demirag and J.M. Leung, A tabu search heuristic for the heterogeneous vehicle routing problem on a multigraph. Transp. Res. Part E: Logistics Transp. Rev. 86 (2016) 32-52.

[14] G. Lera-Romero, J.J. Miranda Bront and F.J. Soulignac, Linear edge costs and labeling algorithms: the case of the timedependent vehicle routing problem with time windows. Networks 76 (2020) 24-53.

[15] C. Liu, G. Kou, X. Zhou, Y. Peng, H. Sheng and F.E. Alsaadi, Time-dependent vehicle routing problem with time windows of city logistics with a congestion avoidance approach. Knowl.-Based Syst. 188 (2020) 104813.

[16] S. Mancini, A combined multistart random constructive heuristic and set partitioning based formulation for the vehicle routing problem with time dependent travel times. Comput. Oper. Res. 88 (2017) 290-296.

[17] M. Mourgaya and F. Vanderbeck, The periodic vehicle routing problem: classification and heuristic. RAIRO:OR 40 (2006) 169-194.

[18] P.K. Nguyen, T.G. Crainic and M. Toulouse, A tabu search for time-dependent multi-zone multi-trip vehicle routing problem with time windows. Eur. J. Oper. Res. 231 (2013) 43-56.

[19] M. Okude and E. Taniguchi, Hierarchical traffic network for heuristic approximation method of vehicle routing problems. Proc.-Soc. Behav. Sci. 125 (2014) 262-274. 
[20] J. Respen, N. Zufferey and J.Y. Potvin, Impact of vehicle tracking on a routing problem with dynamic travel times. RAIRO:OR 53 (2019) 401-414.

[21] N.R. Sabar, A. Bhaskar, E. Chung, A. Turky and A. Song, A self-adaptive evolutionary algorithm for dynamic vehicle routing problems with traffic congestion. Swarm Evol. Comput. 44 (2019) 1018-1027.

[22] M. Setak, M. Habibi, H. Karimi and M. Abedzadeh, A time-dependent vehicle routing problem in multigraph with FIFO property. J. Manuf. Syst. 35 (2015) 37-45.

[23] H. Seyyedhasani and J.S. Dvorak, Using the Vehicle Routing Problem to reduce field completion times with multiple machines. Comput. Electron. Agric. 134 (2017) 142-150.

[24] M. Soysal and M. Çimen, A simulation based restricted dynamic programming approach for the green time dependent vehicle routing problem. Comput. Oper. Res. 88 (2017) 297-305.

[25] R. Spliet, S. Dabia and T. Van Woensel, The time window assignment vehicle routing problem with time-dependent travel times. Transp. Sci. 52 (2018) 261-276.

[26] J. Sun and H.X. Liu, Stochastic eco-routing in a signalized traffic network. Transp. Res. Part C: Emerging Technol. 59 (2015) $32-47$.

[27] Y. Sun, D. Wang, M. Lang and X. Zhou, Solving the time-dependent multi-trip vehicle routing problem with time windows and an improved travel speed model by a hybrid solution algorithm. Cluster Comput. 22 (2019) 15459-15470.

[28] C. Verbeeck, P. Vansteenwegen and E.H. Aghezzaf, Solving the stochastic time-dependent orienteering problem with time windows. Eur. J. Oper. Res. 255 (2016) 699-718.

[29] J.F. Victoria, H.M. Afsar and Ch. Prins, Column generation-based heuristic for the vehicle routing problem with timedependent demand. IFAC Conf. Manuf. Modell. Manage. Control 49 (2016) 526-531.

[30] Z. Xu, A. Elomri, S. Pokharel and F. Mutlu, A model for capacitated green vehicle routing problem with the time-varying vehicle speed and soft time windows. Comput. Ind. Eng. 137 (2019) 106011.

[31] R. Zhang, J. Guo and J. Wang, A Time-dependent electric vehicle routing problem with congestion tolls. IEEE Trans. Eng. Manage. (2020)

[32] J. Zheng, A vehicle routing problem model with multiple fuzzy windows based on time-varying traffic flow. IEEE Access 8 (2020) 39439-39444.

\section{Subscribe to Open (S2O) A fair and sustainable open access model}

This journal is currently published in open access with no charge for authors under a Subscribe-to-Open model (S2O). Open access is the free, immediate, online availability of research articles combined with the rights to use these articles fully in the digital environment.

$\mathrm{S} 2 \mathrm{O}$ is one of the transformative models that aim to move subscription journals to open access. Every year, as long as the minimum amount of subscriptions necessary to sustain the publication of the journal is attained, the content for the year is published in open access.

\section{Ask your library to support open access by subscribing to this S2O journal.}

Please help to maintain this journal in open access! Encourage your library to subscribe or verify its subscription by contacting subscribers@edpsciences.org

We are thankful to our subscribers and sponsors for making it possible to publish the journal in open access, free of charge for authors. More information and list of sponsors: https://www.edpsciences.org/en/maths-s2o-programme 